\title{
Test and Analysis of Gearbox Dedicated Spreader for Cars Workshop
}

\author{
Wang Weixiong ${ }^{1, a}$, Chen Min ${ }^{1, b}$, Huang Guojian ${ }^{1, c}$, Liu Jin ${ }^{1, d}$ \\ ${ }^{1}$ Guangzhou Academy of Special Equipment Inspection \& Testing, Guangzhou Guangdong \\ 510663, China \\ a3136944@qq.com, bzidane2006@qq.com, guojian.huang@gmail.com, d474026743@qq.com
}

Keywords: Finite element analysis; Gearbox spreader; NDT.

Abstract: Automobile assembly workshop has complex process; it usually requires the use of special spreader. Due to highly intensive work, the security requirements of the job are always in high demand, so safety and reliability of the spreader is essential. In this paper, finite element analysis and NDT were used to analyze the spreader, according to the simulation analysis and testing, the stress distribution of the spreader is reasonable, but parts of cracks founded in hook surface. After a comprehensive analysis, the hook needs to be replaced, and better to have regular damage detection.

\section{Introduction}

In recent years, with the rapid development of Chinese industry, automotive industry has become a pillar industry of national economy. In automobile production, final assembly is the most important step. It needs to lift various components for final assembly, usually requires the use of dedicated spreader to lift and transport assembly [1]. Hoisting gearbox is one of key links, and it uses a dedicated spreader for the particularity of the gearbox structure. Due to the highly intensive workshop production line, it requires extremely high job security job security is extremely demanding, and therefore, the safety and reliability of the spreader is crucial [2]. In this paper, finite element simulation and NDT methods are used for analyzing a dedicated spreader of gearbox, and improve the safety of production operations [3].

\section{Basic technical performance testing}

In assembly shop of automobile production line, the dedicated spreader had been manufactured for 10 years, by the calculations, the spreaders work 14 hours and hoist 800 gearboxes a day, and the gearbox weighs about $0.1 \mathrm{~kg}$. The spreader is shown in Fig.1, there are composed of fixed spreader and the detachable hook.

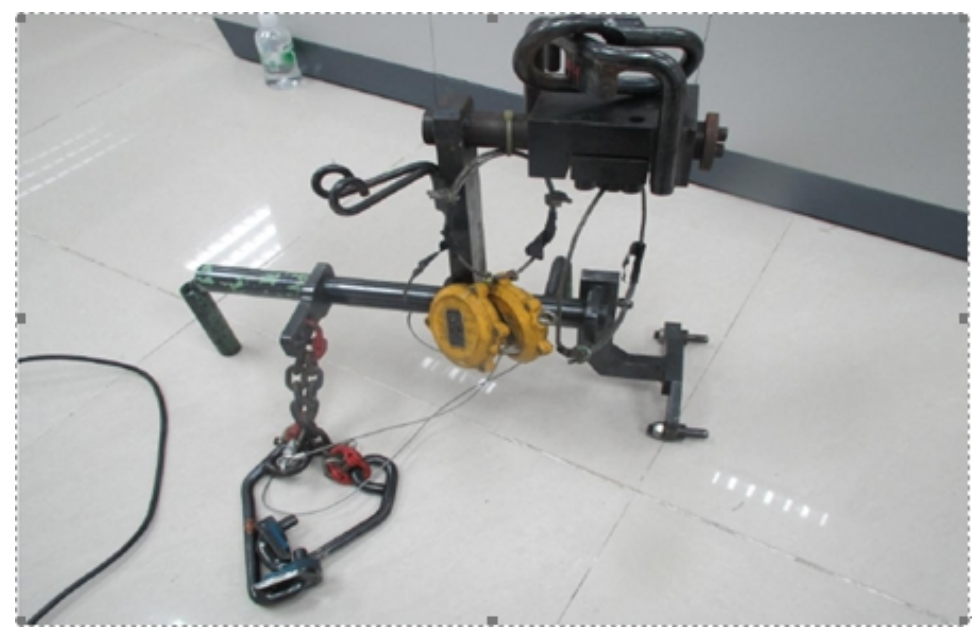

Fig.1 Structure diagram of spreader 
Before further testing, for the sake of the overall analysis and evaluation, it was necessary to inspect the spreaders maintenance records and its morphological appearance and the corrosion, detect the wear of structural metal surface, observe the weld quality, and clarify its basic performance.

(1) Appearance inspection. Check the usage records and maintenance records that provided by the manufacturer. Upon verification, the overall appearances of the spreader were in good condition, and there were no significant structural deformation and damage.

(2) Measurement of Shape \& position. Detect the wear of spreader by using ultrasonic thickness method. The test was carried out under the condition of normal temperature and humidity, and ultrasonic thickness measuring instrument was used for testing. After testing, the anti-corrosion measures of spreader and hook's surface was in good condition, and no abnormal wear found. The test process is shown in Fig.2 Fig.5.

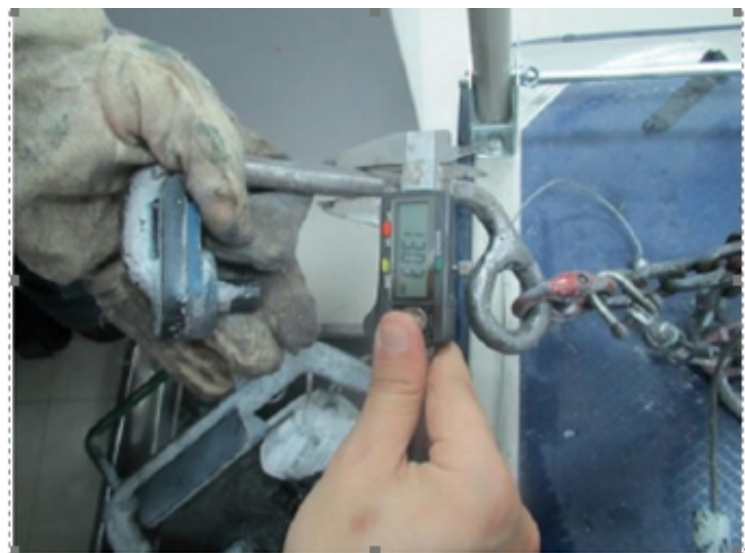

Fig. 2 Spreader thickness test

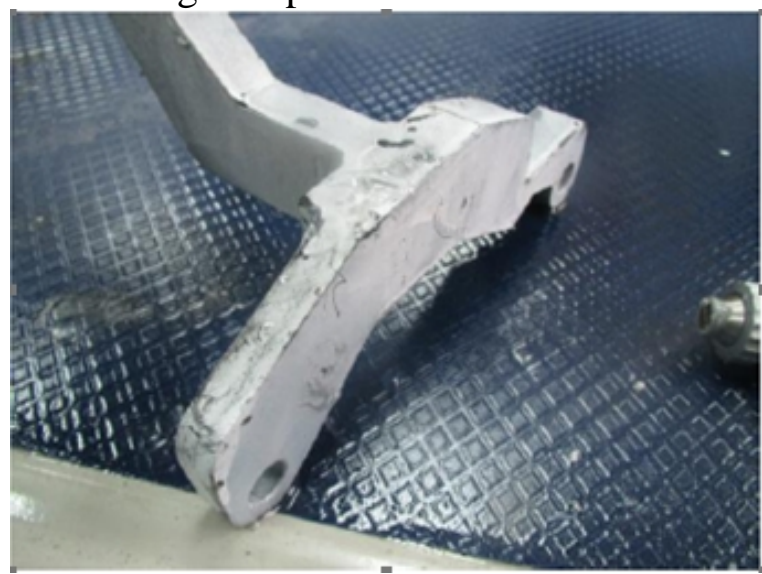

Fig.4 Spreader thickness test

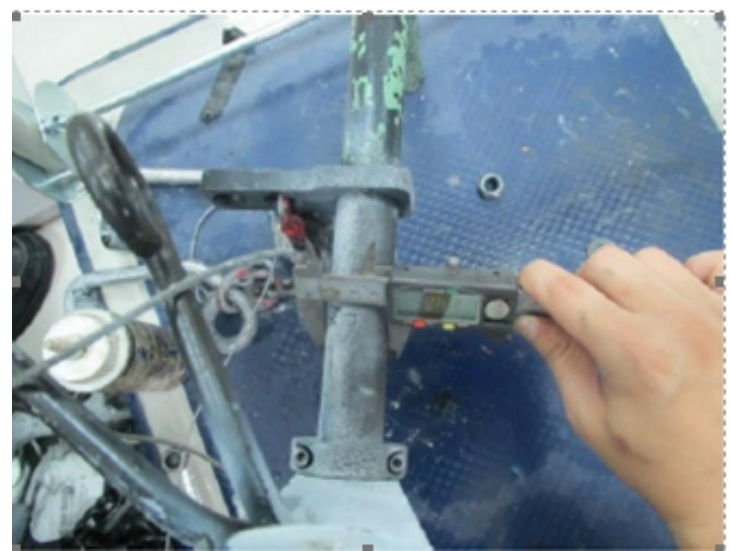

Fig.3 Spreader thickness test

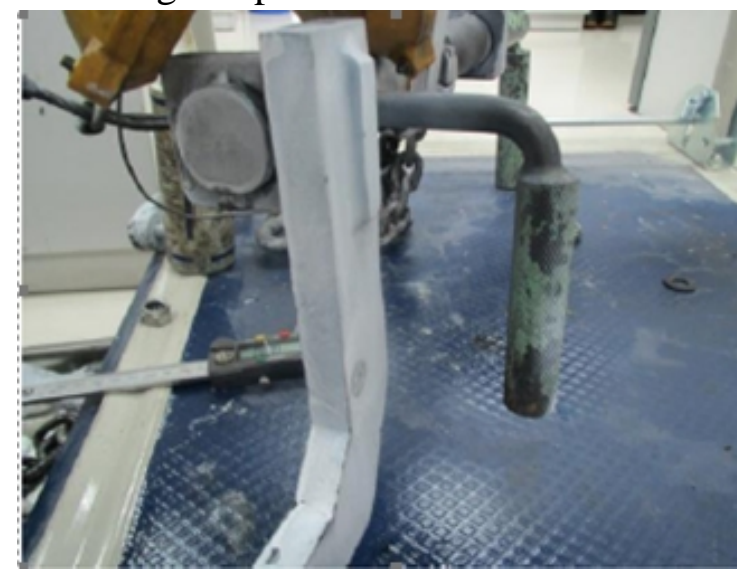

Fig.5 Spreader thickness test

\section{Finite Element Analysis on Structure}

\section{The establishment of finite element model}

The static analysis of the spreader structure is by using finite element method, which can check its strength and stiffness. According to the structural characteristics of the spreader, using Solidworks to build three-dimensional model, and then import it into finite element analysis software for analysis. The following operation should be carried out before the model is calculated:

(1) Material properties setting. The spreader material is Q235 carbon structural steel, which density is $7.85^{*} 10-6 \mathrm{~kg} / \mathrm{mm} 3$, yield limit is $235 \mathrm{MPa}$, elastic modulus of the material is $2.1 * 105 \mathrm{MPa}$, and the Poisson's ratio is 0.3 . All of above are set in the software.

(2) Element type setting. The spreader working mode can be divided into vertical lifting and horizontal transportation. According to the load characteristic, the three-dimensional element is selected as the finite element type. This kind of element has the ability of stretching, compression, torsion and bending, and can consider the effect of shear deformation. 
(3) Meshing. In order to better accuracy, the grid type should be set as hexahedral; the finite element model is shown in Fig.6.

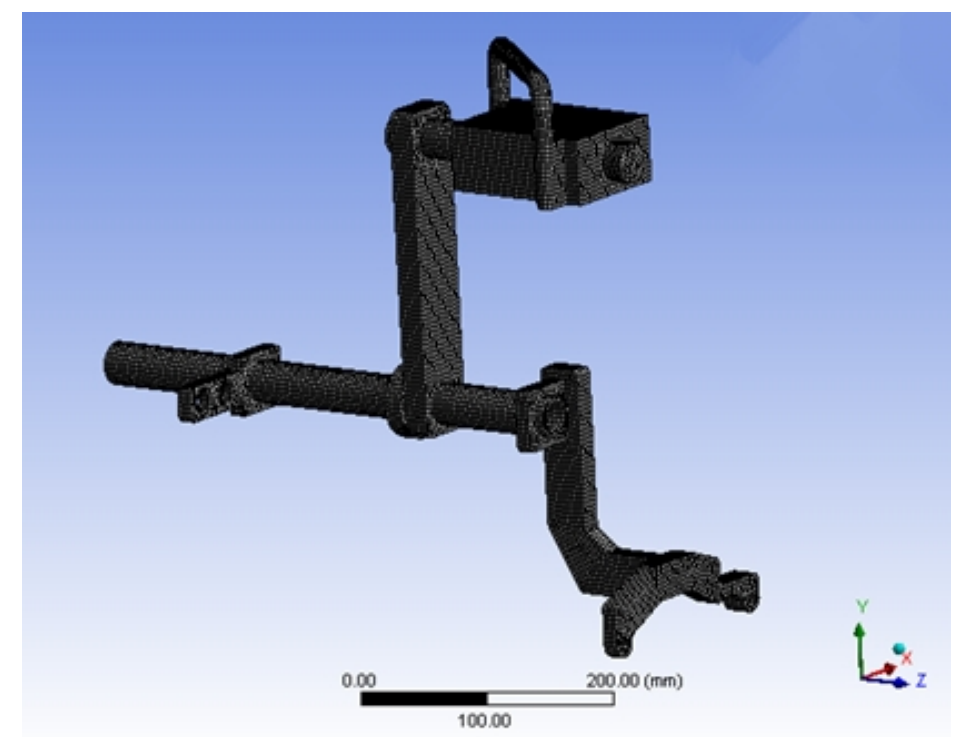

Fig.6 Finite element model

(4) Constraint adding. The spreader was supported by electric hoist pulling, and according to the characteristics, at the center of the upper ring was the constraint position, which applied 6 full freedom constraints.

(5) Applying loads. The spreader worked by inserting inserted two bolts into the corresponding position on gearbox. Therefore, the surface pressure was applied to the load on the bolt hole. In addition, according to the principle of moment balance, the surface pressure was applied on the through-hole which bounded by hook to reach equilibrium.

\section{Result analysis}

Through the calculation of the finite element software, the maximum equivalent stress of the spreader structure appears on the position that the horizontal axis and the vertical piece connections, the value is 95.8 MPa. Stress distribution is shown in Figure 7 and figure 8. Figure 7 and Figure 8 show that the spreader overall stress is not large and distributed evenly. The stress value is generally below $50 \mathrm{MPa}$, and the relative large stress appears on the position of structure discontinuities.

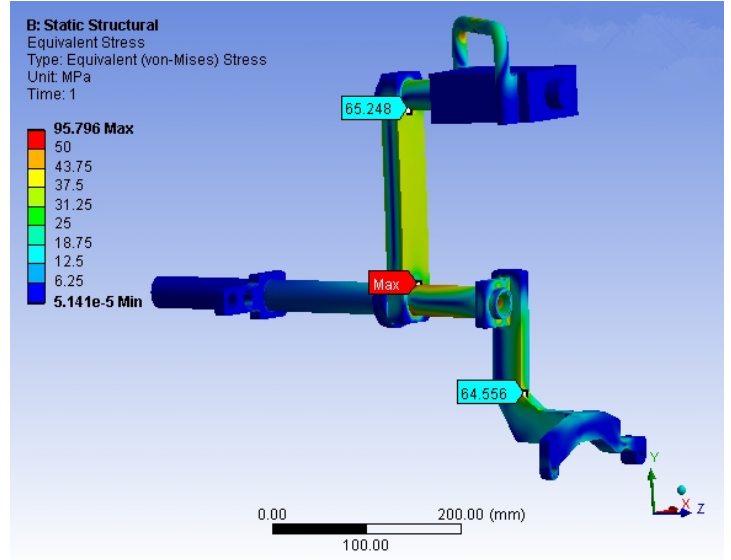

Fig.7 Equivalent stress nephogram

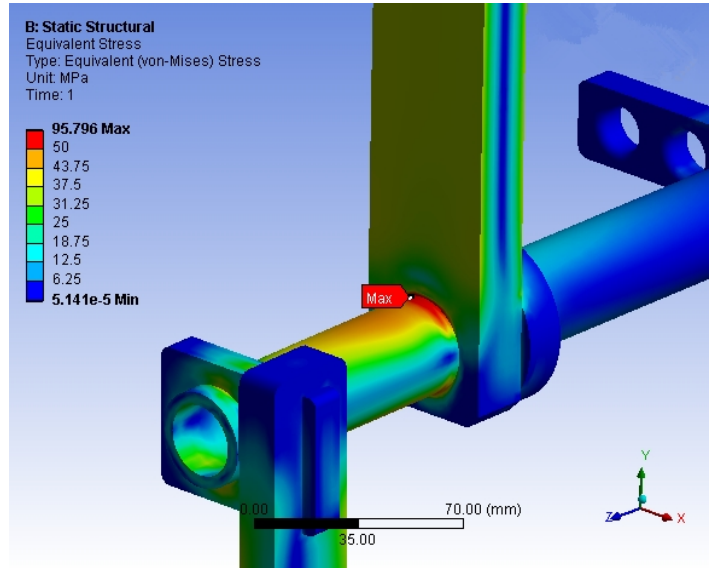

Fig.8 Equivalent stress partially enlarged view 


\section{Magnetic particle testing}

In order to evaluate the performance of dedicated spreader, using non-destructive testing methods to test and analyze it. Magnetic particle testing can detect surface defects of the magnetic material, including cracks, inclusions, hairline, white point, folding, cold shut and alienation, etc. And compared with other nondestructive methods, Magnetic particle testing is simple, flexible and convenient, high sensitive, reliable, non-toxic for human body, non-pollution, etc. [4]

According to the situation, using the magnetic yoke method to detect the spreader all welds and surface inspection by magnetic yoke instrument. The testing process is strictly in accordance with the process procedures. And checking the sensitivity detection of system for three times with A1-30/100 standard specimen. The detection diagrams were shown in Fig.9 to Fig.12.

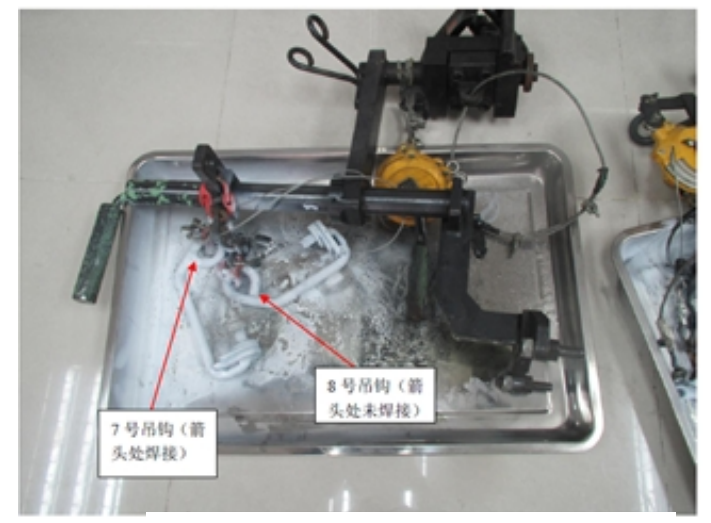

Fig.9 Spreader detection

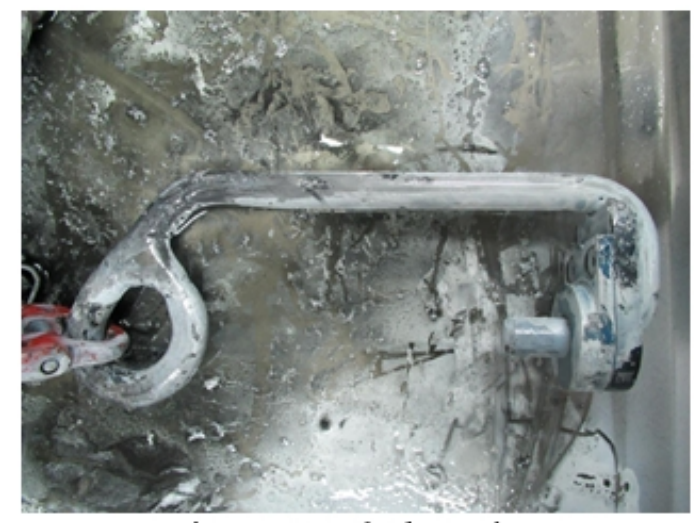

Fig.11 Hook detection

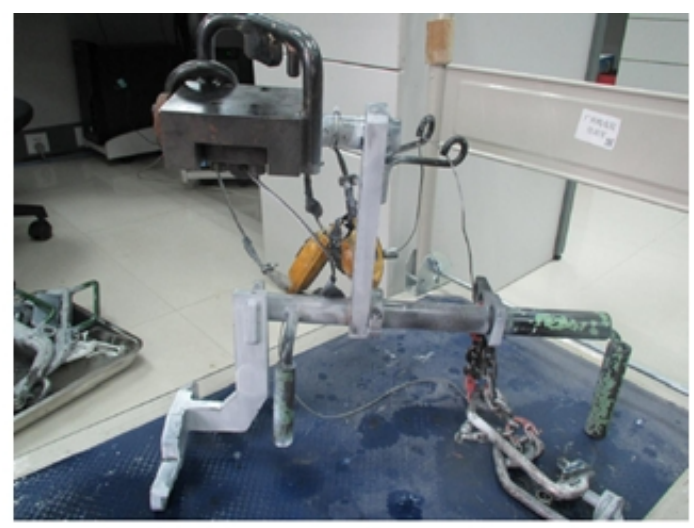

Fig.10 Spreader frame detection

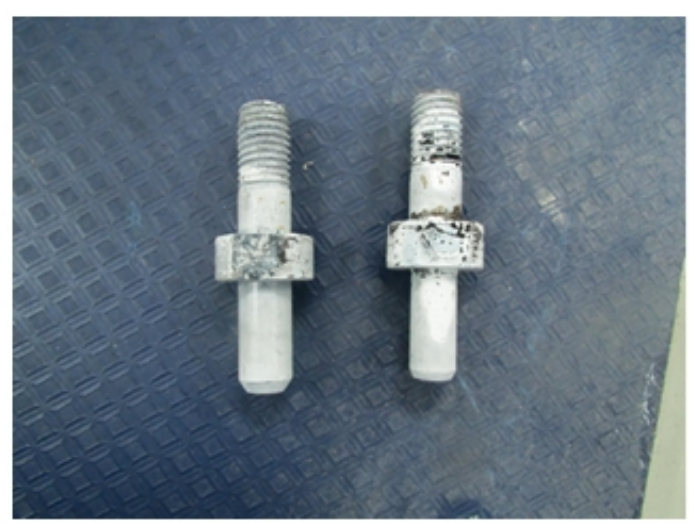

Fig.12 Bolt detection

Magnetic particle testing results show that there are no defects in the spreader main bearing frame and bearing bolt, but the detachable hook the exist crack defects in the key position, as shown in Fig.13 to Fig.14.

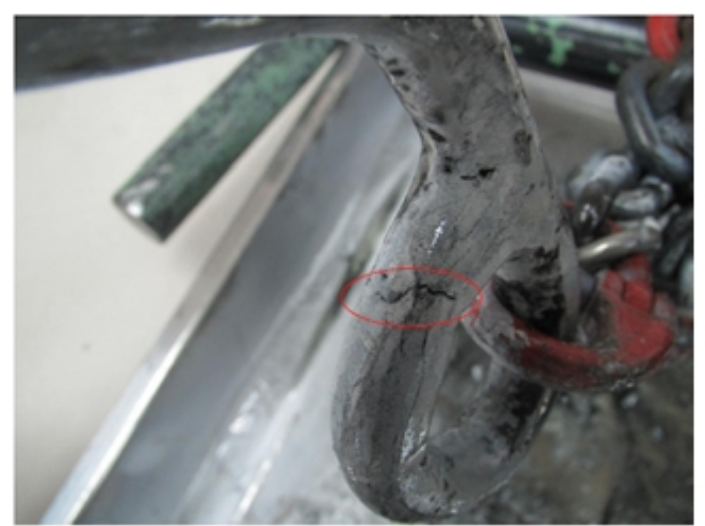

Fig.13 Hook crack

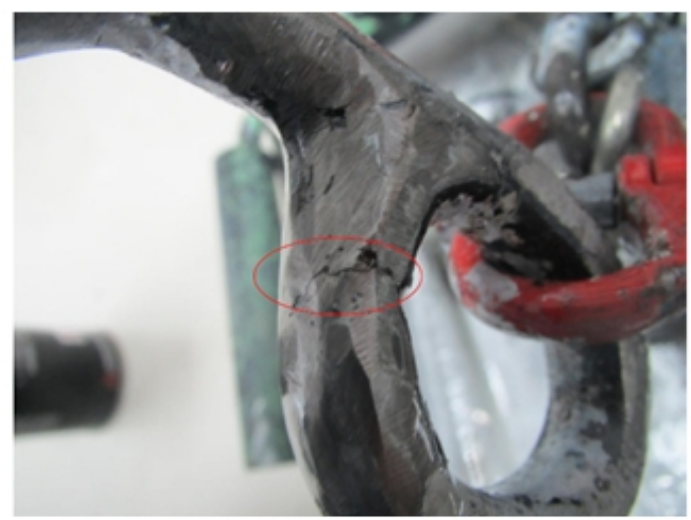

Fig.14 Hook crack 


\section{Conclusions}

Through the finite element analysis, thickness measurement and magnetic particle testing, it shows that the structural static strength of the dedicated spreader meet the requirements during hoisting operation. However, $\mathrm{t}$ crack defects founded in detachable hook, and they need to be replaced to meet the safety requirements. Due to the batch of spreader work over 10 years, they will be better ensuring the safety of lifting operations if they are conducted comprehensive structural damage detection.

\section{Acknowledgements}

This work was financially supported by Technology Projects of Guangdong Provincial Quality and Technical Supervision (2014PT02), Technology Projects of State Administration of Work Safety (Guangdong- 0012-2015AQ), Guangzhou Innovation Platform and Sharing Special Projects (201509010008), Pearl River Technology Nova Special Projects (2013075), Technology Projects of Special Equipment Technology Collaboration Platform (2014SEK003 、2014SEK002 、 2014SEK001).

\section{References}

[1] Wang A J. Simulation and optimization of automobile assembly line [D].Beijing: Beijing Jiaotong University, 2012.

[2] Cai M X, Wang J.Y. Automobile assembly line simulation and optimization [J]. Mechanical design and manufacturing, 2011,11:22-23.

[3] Zhang Y L, Zhang H C. Review of non destructive testing of high end mechanical equipment [J]. Journal of Mechanical Engineering, 2013,49(7):81-87.

[4] Zhou Y Y. Magnetic particle inspection technology of welding seam [J]. Mining machinery. 2007,35(10):141-143.

[5] Zhang Y E. Special lifting slings - Safety Testing balance beam [J]. Hoisting and Conveying Machinery. 2007,1:52-54.

[6] Zhang H. Parametric design and finite element analysis of large equipment special hanger [J]. Hoisting and Conveying Machinery. 2009(6):60-64.

[7] Huang G J. A Modal Analysis of Giant Shipbuilding Tower Crane[J]. Applied Mechanics and Materials.2012, 233(240):473-477.

[8] Qi K. Safety Assessment and Fatigue Life analysis of Aged Crane Structures[C]. the 13th international conference on fracture. 2013:782-787.

[9] Huang, G J .Structural health monitoring of gantry crane based on EDGE technology [J]. Applied Mechanics and Materials. 2013, 333(3): 1629-1634.

[10]Wang W X. Safety assessment technology system for structure of large crane in service [J]. China Special Equipment Safety. 2012, 28(12):12-13. 